\title{
ELEMENTARY ABELIAN 2-GROUPS THAT ACT FREELY ON PRODUCTS OF REAL PROJECTIVE SPACES
}

\author{
LARRY W. CUSICK
}

\begin{abstract}
For a natural number $N$ let $\bar{N}$ be 0 if $N$ is even and 1 if $N$ is odd. We prove that if $\left(\mathbf{Z}_{2}\right)^{\prime}$ acts freely on $\Pi_{1}^{k}=1, \mathbf{R} P^{N_{1}}$ in such a way that the induced action on mod 2 cohomology is trivial. then $l \leqslant 2\left(\bar{N}_{1}+\cdots+\bar{N}_{h}\right)$. If no $N$, is congruent to $3 \bmod 4$ then $l \leqslant \bar{N}_{1}+\cdots+\bar{N}_{h}$
\end{abstract}

In this note we adapt some techniques developed by G. Carlsson [1] to prove certain restrictions on elementary abelian 2 -groups that may act freely on products of real projective spaces.

For $m$ a natural number, let

$$
\bar{m}= \begin{cases}0, & m \text { even } \\ 1, & m \text { odd }\end{cases}
$$

MaIn Theorem. Suppose $X$ is a finite $C W$-complex with the mod 2 cohomology of $\Pi_{1=1}^{k} \mathbf{R} P^{i}$. Suppose further that $X$ admits a free action by $\mathbf{Z}_{2}^{\prime}$ such that the induced action on mod 2 cohomology is trivial. Then (a) $l \leqslant 2\left(\bar{N}_{1}+\cdots+\bar{N}_{h}\right)$, and (b) if no $N$, is congruent to $3 \bmod 4$ then $l \leqslant \bar{N}_{1}+\cdots+\bar{N}_{k}$.

We would like to remark on the sharpness of the above theorem. It is known that any $\mathbf{R} P^{2 n+1}$ admits a free $\mathbf{Z}_{2}$-action and $\mathbf{R} P^{4 n+3}$ a free $\mathbf{Z}_{2}^{2}$-action (the latter arises from the free quaternion action on $S^{4 n+3}$ ). Thus, if each $N$, is even or congruent to $3 \bmod 4$, then $\Pi_{,=1}^{k} \mathbf{R} P^{N_{1}}$ will admit a free $\mathbf{Z}_{2}^{2\left(N_{1}+\cdots+N_{k}\right)}$-action satisfying the above

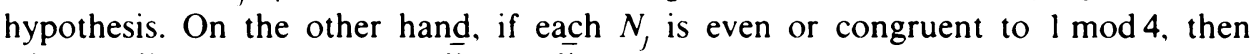
$\Pi_{j=1}^{k} \mathbf{R} P^{N_{i}}$ will admit a free $\mathbf{Z}_{2}^{\bar{N}_{1}}+\cdots+\bar{N}_{h_{-}}$action.

In light of all this, there seems to be an obvious conjecture that is compatible with the above theorem. To state the conjecture we define a function $\mu$ on natural numbers by

$$
\mu(m)= \begin{cases}0 & \text { if } m \text { even } \\ 1 & \text { if } m \equiv 1 \bmod 4 \\ 2 & \text { if } m \equiv 3 \bmod 4\end{cases}
$$

CONJECTURE. Suppose $X$ and $\mathbf{Z}_{2}^{\prime}$ satisfy the hypothesis of the main theorem; then $l \leqslant \mu\left(N_{1}\right)+\cdots+\mu\left(N_{k}\right)$.

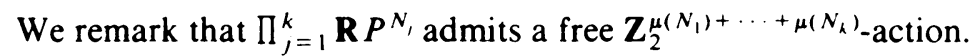

Received by the editors June 28, 1982

1980 Mathematics Subject Classification. Primary 57S17, 55S07: Secondary 55R25, 55T10 
Suppose $X$ is a finite CW-complex that admits an action by a group $G$. Let $X_{G}$ denote $E G \times{ }_{G} X$. Consider the mod 2 Serre spectral sequence associated to the fibration $X \rightarrow X_{G} \rightarrow B G$. It has $E_{2}$-term

$$
E_{2}^{* *} \cong H^{*}\left(B G ; H^{*}\left(X ; \mathbf{Z}_{2}\right)\right) \text {. }
$$

Proposition A. If $G$ acts freely on $X$ then $E_{\infty}$ is a finite-dimensional $\mathbf{Z}_{2}$-vector space.

Proof. Under the hypothesis $E G \times_{G} X \simeq X / G$, which is a finite CW-complex. The proposition follows since $E_{\infty}^{* *}$ is the associated graded groups to some filtration on $H^{*}\left(X / G ; \mathbf{Z}_{2}\right)$ which is finite dimensional.

Now assume that $X$ and $G=\mathbf{Z}_{2}^{l}$ satisfy the hypothesis of the Main Theorem, and consider the Serre spectral sequence associated to the fibration

$$
X \rightarrow X_{G} \rightarrow B G \text {. }
$$

Our $E_{2}$ term looks like

$$
\begin{aligned}
E_{2}^{* *} & \cong H^{*}\left(B G ; H^{*}\left(X ; \mathbf{Z}_{2}\right)\right) \\
& \cong H^{*}\left(B G ; \mathbf{Z}_{2}\right) \otimes H^{*}\left(X ; \mathbf{Z}_{2}\right) \\
& \cong \mathbf{Z}_{2}\left[\gamma_{1}, \ldots, \gamma_{l}\right] \otimes \mathbf{Z}_{2}\left[Z_{1}, \ldots, Z_{k}\right] /\left(Z_{1}^{N_{1}+1} \ldots \ldots Z_{h}^{N_{h}+1}\right),
\end{aligned}
$$

where $\operatorname{dim} \gamma_{1}=1$ and $\operatorname{dim} Z_{J}=1$.

Let $w_{j}=d_{2}\left(Z_{j}\right) \in H^{2}\left(B G ; \mathbf{Z}_{2}\right)$, where $d_{2}$ is the first differential in the spectral sequence.

Proposition B. (a) If $N_{j}$ is even, then $w_{j}=0$, and (b) if $N_{j} \equiv 1 \bmod 4$, then $\mathrm{Sq}^{1} w_{j}$ is an element of the ideal $\left(w_{1}, \ldots, w_{k}\right)$ in $H^{*}\left(B G ; \mathbf{Z}_{2}\right)$.

Proof. (a) Assume $N_{j}=2 m$. Then $0=d_{2}\left(Z_{1}^{2 m+1}\right)=Z_{1}^{2 m} \otimes d_{2}\left(Z_{j}\right)$. since $d_{2}$ is a derivation. Now we just note that the map

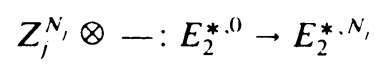

is an injection, so $d_{2}\left(Z_{j}\right)=0$.

(b) Now assume $N_{j}=4 m+1$. We again use the derivation property of the differentials to compute $0=d_{3}\left(Z_{j}^{4 m+2}\right)=Z_{j}^{4 m} \cdot d_{3}\left(Z_{j}^{2}\right)$. Now $Z_{j}^{2}=\mathrm{Sq}^{1} Z$, and the transgression operator commutes with the action of the Steenrod algebra, so $d_{3}\left(Z_{1}^{2}\right)$ is represented by $\mathrm{Sq}^{1} w_{j}$, and so, we must have that $Z_{j}^{4 m} \otimes \mathrm{Sq}^{1} w_{1} \in E_{2}^{3.4 m}$ is in the image of $d_{2}$. But $d_{2}$ is completely determined by $d_{2}\left(Z_{j}\right)=w_{j}$ and the fact that it is a derivation. So we must have

$$
Z_{j}^{4 m} \otimes \mathrm{Sq}^{1} w_{j}=Z_{1}^{4 m} \otimes d_{2} f
$$

where $f \in E_{2}^{1.1}$. But $Z_{j}^{4 m} \otimes-: E_{2}^{* .0} \rightarrow E_{2}^{* .4 m}$ is an injection. so $\mathrm{Sq}^{1} w_{1}=d_{2} f$. Now, write $f=\Sigma_{i, j} \lambda_{1} Z_{1} \otimes \gamma_{j}$, where each $\lambda_{i}, \in \mathbf{Z}_{2}$. Then, we compute

$$
d_{2} f=\sum_{i, 1} \lambda_{1}, w_{i} \gamma_{1} \text {, }
$$

which is clearly in the stated ideal. 
Proposition C [1]. Suppose $f_{1}, \ldots, f_{k}$ are elements of $H^{n}\left(B \mathbf{Z}_{2}^{\prime} ; \mathbf{Z}_{2}\right)$, regarded as homogeneous polynomials of degree $n$ in $l$ variables. Then they have a nontrivial common zero in $\mathbf{Z}_{2}^{\prime}$ if and only if there is a subgroup inclusion $i: \mathbf{Z}_{2} \hookrightarrow \mathbf{Z}_{2}^{\prime}$ such that each $i^{*} f_{j}=0$.

PROPOSITION D [3]. If $f_{1}, \ldots, f_{k}$ are homogeneous polynomials in l variables of degree $n$ with coefficients in $\mathbf{Z}_{2}$, and if $l>n k$, then they have a nontrivial common zero in $\mathbf{Z}_{2}^{\prime}$.

Proof of Main Theorem. Part (a). We first claim that the degree 2 polynomials $w_{1}, \ldots, w_{k}$ have no nontrivial common zero. If they did, then Proposition $\mathrm{C}$ would provide a subgroup inclusion $i: \mathbf{Z}_{2} \hookrightarrow \mathbf{Z}_{2}^{l}$ for which each $i^{*} w_{j}=0$. But if this were the case, we could restrict our $G$-action on $X$ to this $\mathbf{Z}_{2}$, and the naturality of the Serre spectral sequence with respect to subgroup inclusions would imply that the sequence for the fibration $X \rightarrow X_{\mathbf{Z}_{2}} \rightarrow B \mathbf{Z}_{2}$ would collapse, $E_{2}=E_{x}$. which is impossible since, by Proposition A, $E_{x}$ must be finite dimensional. Since there can be no such restriction there can be no nontrivial common zero.

Part (a) of the Main Theorem now follows from Propositions D and B. part (a).

Proposition E [1]. Let $\left(f_{1} \ldots \ldots f_{h}\right)$ be an ideal generated by homogeneous polynomials $f_{1}$ in $\mathbf{Z}_{2}\left[\gamma_{1} \ldots, \gamma_{1}\right]$ which is invariant under the action of the Steenrod algebra. Then if $l>k$, there exists a nontrivial common zero to $f_{1} \ldots \ldots f_{h}$.

Proof of Main Theorem. Part (b). The hypothesis and Proposition B imply that the ideal $\left(n_{1}, \ldots, n_{h}\right)$ is invariant under the action of the Steenrod algebra. Proposition $\mathrm{E}$ implies that if $l>\bar{N}_{1}+\cdots+\bar{N}_{h}$, then there would exists a nontrivial common zero to $n_{1}, \ldots, w_{h}$. But we have already seen. in the proof of part (a), that this is an impossibility. Thus, we must have $l \leqslant \bar{N}_{1}+\cdots+\bar{N}_{h}$.

\section{BIBLIOGRAPHY}

1. (i. Carlsson. On the non-existence of free actions of elementary ahelian groups on products of spheres. Amer. J. Math. 102 (1980). 1147-1157.

2. On the ranh of abelian groups actung freely on $\left(S^{\prime \prime}\right)^{h}$. Invent. Math. (to appear)

3. M. Cireenberg. Lectures on forms in mam cariables. Benjamin. Neu York. 1969.

Department of Mathematics. California State University. Long Beach. Cal.ifornia 90840 